\title{
Lifshitz-like solutions with hyperscaling violation in ungauged supergravity
}

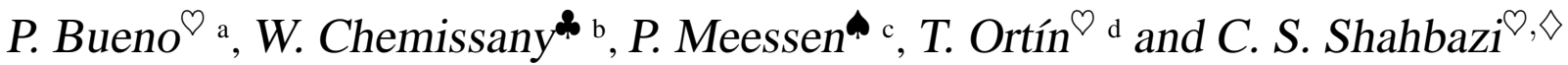 \\ ${ }^{\circ}$ Instituto de Física Teórica UAM/CSIC \\ C/ Nicolás Cabrera, 13-15, C.U. Cantoblanco, 28049 Madrid, Spain \\ *Department of Physics and Astronomy, University of Waterloo, Waterloo, \\ Ontario, Canada, N2L $3 G 1$ \\ "HEP Theory Group, Departamento de Física, Universidad de Oviedo \\ Avda. Calvo Sotelo s/n, 33007 Oviedo, Spain \\ $\diamond$ Stanford Institute for Theoretical Physics and Department of Physics, Stanford University, \\ Stanford, CA 94305-4060, USA
}

\begin{abstract}
In this note we describe several procedures to construct, from known black-hole and black-brane solutions of any ungauged supergravity theory, non-trivial gravitational solutions whose "near-horizon" and "near-singularity" limits are Lifshitz-like spacetimes with dynamical critical exponent $z$, "hyperscaling violation" exponent $\theta$ and Lifshitz radius $\ell$ that depends on the physical parameters of the original black-hole solution. Since the new Lifshitz-like solutions can be constructed from any black-hole solution of any ungauged supergravity, many of them can be easily embedded in String Theory. Some of the procedures produce supersymmetric Lifshitz-like solutions.
\end{abstract}

\footnotetext{
${ }^{a}$ E-mail: pab.bueno [at] estudiante.uam.es

${ }^{b}$ E-mail: chemissany.wissam [at] gmail.com

${ }^{c}$ E-mail: meessenpatrick [at] uniovi.es

${ }^{\mathrm{d}}$ E-mail: Tomas.Ortin [at] csic.es

${ }^{e}$ E-mail: Carlos.Shabazi [at] uam.es
} 


\section{Contents}

1 Introduction $\quad 2$

2 The generalized FGK formalism 3

3 Solutions with Lifshitz-like asymptotics 7

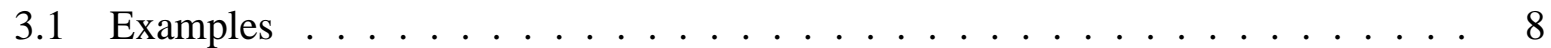

4 More hvLf metrics 11

4.1 hvLf spaces from limiting procedures . . . . . . . . . . . . . . 11

4.2 Supersymmetric hvLf spaces from smearing . . . . . . . . . . . . . . 11

4.3 Higher dimensional generalization . . . . . . . . . . . . . . . . . . . 14

5 Discussion 16

A Some properties of the hvLf metrics 17

\section{Introduction}

Gauge/gravity duality has found new and interesting applications in the study of strongly coupled condensed matter systems [1, 2, 3]. In this context one has to work with the metrics that are dual to scale-covariant field theories which are not conformally invariant. These theories are characterized by a dynamical critical exponent $z \neq 1$ and a hyperscaling violation exponent $\theta \neq 0$ [4, 5, 6, 7]. The values $z=1$ and $\theta=0$ correspond to conformally-invariant theories dual to the AdS metric. For other values of $z$ and $\theta$, in terms of dimensionless coordinates $t, r, x^{i}$, the $(d+2)$-dimensional spacetime metric can be cast in the form

$$
d s_{d+2}^{2}=\ell^{2} r^{-2(d-\theta) / d}\left[r^{-2(z-1)} d t^{2}-d r^{2}-d x^{i} d x^{i}\right],
$$

where $d$ is the number of spatial dimensions on which the dual theory lives $(i=1, \ldots, d)$ and the parameter $\ell$, with dimensions of length, is the Lifshitz radius. We will refer, henceforth, to these metrics as hyperscaling-violating Lifshitz $(h v L f)$ metrics.

hvLf geometries (1.1) with the particular hyperscaling violation exponent $\theta=d-1$ are intimately connected with compressible states with hidden Fermi surfaces as well as with logarithmic violations of the area law of entanglement entropy (see for instance [5, 6, 7, 8]). hvLf solutions have also been of interest for their connection with string theory and supergravity. We refer the reader to $e . g$. [6, 9, 10, 11] .

It is known since the advent of the AdS/CFT duality that considering temperature in the gauge theory corresponds to putting a black hole in the bulk of the gravity side [12, 13]. When the gauge theory is conformal, the corresponding black hole has AdS asymptotics in the boundary. Similarly, since Lifshitz field theory with hyperscaling violation has anisotropic scale covariance, the black holes describing the geometry dual to its finite temperature generalization must have a metric of the form (1.1) as asymptotic geometry. 
Asymptotically Lifshitz black holes with $\theta=0$ and $z \neq 1$ have been extensively studied over the last few years. Analytic and numerical solutions to gravitational theories with simple types of matter have been constructed. String theoretic black hole solutions having Lifshitz asymptotics with $\theta=0$ with a general dynamical exponent have been numerically computed (see [14, 15] and references therein). Analytical black hole solutions which could be related to string and supergravity theories are still missing, though 1

So far, hvLf metrics (1.1) with $\theta \neq 0$ have only been found in solutions to Einstein-Maxwelldilaton-type effective actions of the form

$$
S=\frac{1}{16 \pi G_{N}} \int \sqrt{|g|}\left\{R+\frac{1}{2} \partial_{\mu} \phi \partial^{\mu} \phi-Z(\phi) F^{\mu \nu} F_{\mu \nu}-2 \Lambda-V(\phi)\right\} .
$$

Simple analytic black hole solutions have been constructed for this model for specific choices of $V(\phi)$ and $Z(\phi)$ in [5, 6, 7, 8, 17]; analytical hvLf solutions to a model with 2 gauge fields and an exponential scalar potential, are presented and analysed in ref. [38]. Finding embeddings of these models and solutions in gauged supergravities and, eventually, in string theory would be most interesting, in particular for asymptotically hvLf $\theta=d-1$ black holes.

In this work we report progress in this direction. In particular, we are going to show how to construct systematically solutions of ungauged supergravity whose metrics are, or approach in certain limits, hvLf metrics with certain values of $z$ and $\theta$. The first of our constructions makes use of the FGK formalism originally developed to study static, spherically symmetric, asymptotically flat, black hole solutions of 4-dimensional ungauged supergravity theories [22], and we start by reviewing this formalism in Section 2. We will then generalize the FGK formalism to metrics which are not spherically symmetric. The main result is that there are (at least) two cases in which the equations of motion of the metric function and scalar fields are identical to those of the spherically symmetric one. Thus, one can use the solutions of the standard black hole case and construct solutions with entirely different spacetime metrics.

In section 3 we study the behaviour of the new solutions in the neighborhood of the values of the radial coordinate corresponding, in the original solution, to the inner and outer horizons, spatial infinity and the curvature singularity. We will find hvLf metrics in some of these limits. In Section 4 we investigate how hvLf metrics arise in other limits of more standard metrics and propose other procedures to construct, in particular, supersymmetric hvLf spacetimes by smearing extremal supersymmetric black hole solutions of $N=2, d=4$ supergravity. In Section 3 we briefly discuss the generalization of these results to higher dimensions. A brief discussion of our results can be found in Section 5 and the appendix contains a summary of properties of hvLf metrics.

\section{The generalized FGK formalism}

Following Ref. [22] we consider the action

$$
I=\int d^{4} x \sqrt{|g|}\left\{R+\mathcal{G}_{i j}(\phi) \partial_{\mu} \phi^{i} \partial^{\mu} \phi^{j}++2 \Im \mathrm{m} \mathcal{N}_{\Lambda \Sigma} F^{\Lambda}{ }_{\mu \nu} F^{\Sigma \mu \nu}-2 \Re \mathrm{e} \mathcal{N}_{\Lambda \Sigma} F^{\Lambda}{ }_{\mu \nu} \star F^{\Sigma \mu \nu}\right\}
$$

\footnotetext{
${ }^{1}$ The analytic black hole solutions in [16] suffer from naked singularities.
} 
where $\mathcal{N}_{\Lambda \Sigma}$ is the complex scalar-dependent (period) matrix. The bosonic sector of any ungauged supergravity theory in 4 dimensions can be put in this form. The number of scalars labeled by $i, j, \cdots$ and of vector field labeled by $\Lambda, \Sigma, \cdots$, the scalar metric $\mathcal{G}_{i j}$ and the period matrix $\mathcal{N}_{\Lambda \Sigma}$ depend on the particular theory under consideration.

Since we want to obtain static solutions, we consider the metric

$$
d s^{2}=e^{2 U} d t^{2}-e^{-2 U} \gamma_{\underline{m n}} d x^{\underline{m}} d x^{\underline{n}},
$$

where $\gamma_{\underline{m n}}$ is a 3-dimensional (transverse) Riemannian metric to be specified later. Using Eq. (2.2) and the assumption of staticity of all the fields, we perform a dimensional reduction over time in the equations of motion that follow from the above general action. We obtain a set of reduced equations of motion that we can write in the form 2

$$
\begin{aligned}
\nabla_{\underline{m}}\left(\mathcal{G}_{A B} \partial^{\underline{m}} \tilde{\phi}^{B}\right)-\frac{1}{2} \partial_{A} \mathcal{G}_{B C} \partial_{\underline{m}} \tilde{\phi}^{B} \partial^{\underline{m}} \tilde{\phi}^{C}=0 . \\
R_{\underline{m n}}+\mathcal{G}_{A B} \partial_{\underline{m}} \tilde{\phi}^{A} \partial_{\underline{n}} \tilde{\phi}^{B}=0 . \\
\partial_{[\underline{m}} \psi^{\Lambda} \partial_{\underline{n}]} \chi_{\Lambda}=0,
\end{aligned}
$$

where all the tensor quantities refer to the 3-dimensional metric $\gamma_{\underline{m n}}$ and we have defined the metric $\mathcal{G}_{A B}$

$$
\mathcal{G}_{A B} \equiv\left(\begin{array}{ccc}
2 & & \\
& \mathcal{G}_{i j} & \\
& & 4 e^{-2 U} \mathcal{M}_{M N}
\end{array}\right)
$$

in the extended manifold of coordinates $\tilde{\phi}^{A}=\left(U, \phi^{i}, \psi^{\Lambda}, \chi_{\Lambda}\right)$, where

$$
\left(\mathcal{M}_{M N}\right) \equiv\left(\begin{array}{cr}
\left(\mathfrak{I}+\mathfrak{R} \mathfrak{I}^{-1} \mathfrak{R}\right)_{\Lambda \Sigma} & -\left(\mathfrak{R} \mathfrak{I}^{-1}\right)_{\Lambda}{ }^{\Sigma} \\
-\left(\mathfrak{I}^{-1} \mathfrak{R}\right)^{\Lambda} & \left(\mathfrak{I}^{-1}\right)^{\Lambda \Sigma}
\end{array}\right), \quad \mathfrak{R}_{\Lambda \Sigma} \equiv \Re \mathrm{e} \mathcal{N}_{\Lambda \Sigma}, \quad \mathfrak{I}_{\Lambda \Sigma} \equiv \Im \mathrm{m} \mathcal{N}_{\Lambda \Sigma}
$$

Eqs. (2.3) and (2.4) can be obtained from a three-dimensional effective action

$$
I=\int d^{3} x \sqrt{|\gamma|}\left\{R+\mathcal{G}_{A B} \partial_{\underline{m}} \tilde{\phi}^{A} \partial^{\underline{m}} \tilde{\phi}^{B}\right\},
$$

but we still need to add the constraint Eq. (2.5).

If we now decide to consider spherically-symmetric transverse metrics only, as it is appropriate to describe single, static black holes, we can choose, as in Ref. [22]

$$
\gamma_{\underline{m n}} d x^{\underline{m}} d x^{\underline{n}}=\frac{d \tau^{2}}{W_{-1}^{4}}+\frac{d \Omega_{-1}^{2}}{W_{-1}^{2}}
$$

\footnotetext{
${ }^{2}$ See Ref. [22] for more details on this reduction.
} 
where $W_{-1}$ is a function of the (inverse) radial coordinate $\tau$ to be determined and

$$
d \Omega_{-1}^{2} \equiv d \theta^{2}+\sin ^{2} \theta d \phi^{2},
$$

is the metric of the round 2-sphere of unit radius. With this choice, Eq. (2.5) is automatically solved, the equation of $W_{-1}(\tau)$ can be integrated completely, giving

$$
W_{-1}(\tau)=\frac{\sinh \left(r_{0} \tau\right)}{r_{0}}
$$

and we are left with just

$$
\begin{aligned}
\frac{d}{d \tau}\left(\mathcal{G}_{A B} \frac{d \tilde{\phi}^{B}}{d \tau}\right)-\frac{1}{2} \partial_{A} \mathcal{G}_{B C} \frac{d \tilde{\phi}^{B}}{d \tau} \frac{d \tilde{\phi}^{C}}{d \tau} & =0 \\
\mathcal{G}_{B C} \frac{d \tilde{\phi}^{B}}{d \tau} \frac{d \tilde{\phi}^{C}}{d \tau} & =2 r_{0}^{2} .
\end{aligned}
$$

The integration constant $r_{0}$ is the non-extremality parameter: when $r_{0}$ vanishes, the metric describes extremal black holes (if the solution satisfies the necessary regularity conditions).

The electrostatic and magnetostatic potentials $\psi^{\Lambda}, \chi_{\Lambda}$ only appear through their $\tau$-derivatives. The associated conserved quantities are the magnetic and electric charges $p^{\Lambda}, q_{\Lambda}$ and can be used to eliminate completely the potentials. The remaining equations of motion can be put in the convenient form

$$
\begin{aligned}
U^{\prime \prime}+e^{2 U} V_{\mathrm{bh}} & =0, \\
\left(U^{\prime}\right)^{2}+\frac{1}{2} \mathcal{G}_{i j} \phi^{i \prime} \phi^{j \prime}+e^{2 U} V_{\mathrm{bh}} & =r_{0}^{2}, \\
\left(\mathcal{G}_{i j} \phi^{j \prime}\right)^{\prime}-\frac{1}{2} \partial_{i} \mathcal{G}_{j k} \phi^{j \prime} \phi^{k \prime}+e^{2 U} \partial_{i} V_{\mathrm{bh}} & =0,
\end{aligned}
$$

in which the primes indicate differentiation with respect to $\tau$ and the so-called black-hole potential $V_{\mathrm{bh}}$ is given by

$$
-V_{\mathrm{bh}}(\phi, \mathcal{Q}) \equiv-\frac{1}{2} \mathcal{Q}^{M} \mathcal{Q}^{N} \mathcal{M}_{M N}, \quad\left(\mathcal{Q}^{M}\right) \equiv\left(\begin{array}{c}
p^{\Lambda} \\
q_{\Lambda}
\end{array}\right)
$$

Eqs. (2.14) and (2.16) can be derived from the effective action

$$
I_{\mathrm{eff}}\left[U, \phi^{i}\right]=\int d \tau\left\{\left(U^{\prime}\right)^{2}+\frac{1}{2} \mathcal{G}_{i j} \phi^{i \prime} \phi^{j \prime}-e^{2 U} V_{\mathrm{bh}}\right\}
$$

Eq. (2.15) is nothing but the conservation of the Hamiltonian (due to absence of explicit $\tau$ dependence of the Lagrangian) but with a particular value of the integration constant $\left(r_{0}^{2}\right)$.

\footnotetext{
${ }^{3}$ As in Ref. [23], we adopt the sign of the black-hole potential opposite to most of the literature on black-hole attractors, conforming instead to the conventions of Lagrangian mechanics.
} 
A fair number of solutions of this system for different theories of $\mathcal{N}=2, d=4$ supergravity coupled to vector supermultiplets are known (see e.g. Ref. [23, 24]). They describe single, charged, static, spherically-symmetric, asymptotically-flat, non-extremal black holes which generalize the Reissner-Nordström solution and have two horizons that coincide when the nonextremality parameter $r_{0}$ vanishes. The metric covers the exterior of the outer (event) horizon when the (inverse) radial coordinate $e^{4} \tau$ takes values in the interval $(-\infty, 0)$, whose limits are, respectively, the event horizon and spatial infinity. The interior of the inner (Cauchy) horizon corresponds to the interval $\left(\tau_{\mathrm{s}},+\infty\right)$, whose limits are, respectively, the singularity and the inner horizon.

We may also be interested in spacetime metrics which are not spherically symmetric, in which case we have to use a different transverse metric. In principle, these metrics are not appropriate to describe isolated, static black holes but here we are ultimately interested in Lifshitz metrics with a transverse metric invariant under the 2-dimensional Euclidean group, Thus, we can take, for instance, the following simple generalization of the spherically-symmetric transverse metric Eq. (2.9):

$$
\gamma_{\underline{m n}} d x^{\underline{m}} d x^{\underline{n}}=\frac{d \tau^{2}}{W_{\kappa}^{4}}+\frac{d \Omega_{\kappa}^{2}}{W_{\kappa}^{2}},
$$

where $W_{\kappa}$ is a function of $\tau$ and $d \Omega_{\kappa}^{2}$ is the metric of the 2-dimensional symmetric space of curvature $\kappa$ and unit radius:

$$
\begin{aligned}
d \Omega_{-1}^{2} & \equiv d \theta^{2}+\sin ^{2} \theta d \phi^{2} \\
d \Omega_{+1}^{2} & \equiv d \theta^{2}+\sinh ^{2} \theta d \phi^{2} \\
d \Omega_{0}^{2} & \equiv d \theta^{2}+d \phi^{2}
\end{aligned}
$$

In these three cases the equation for $W_{\kappa}(\tau)$ can be integrated and the results are

$$
\begin{aligned}
W_{-1} & =\frac{\sinh r_{0} \tau}{r_{0}}, \\
W_{1} & =\frac{\cosh r_{0} \tau}{r_{0}}, \\
W_{0}^{ \pm} & =a e^{\mp r_{0} \tau},
\end{aligned}
$$

where $a$ is a real arbitrary constant with dimensions of inverse length.

It turns out that if we follow now for the $\kappa=0,+1$ cases the procedure described above for the $\kappa=-1$ case we arrive to exactly the same system of equations (2.14)-(2.16) and, therefore, to the same effective action Eq. (2.18). It follows that all the solutions for $\left(U, \phi^{i}\right)$ obtained in the spherically-symmetric case $\kappa=-1$ are also solutions for the $\kappa=0,+1$ cases as well. In other

\footnotetext{
${ }^{4}$ Observe that $\tau$ has dimensions of inverse length, since $r_{0}$ has, conventionally, dimensions of length.
} 
words: every solution of the system of equations (2.14)-(2.16) provides us with four different solutions of the original theory, by simply using the four different transverse metrics.

Since, as mentioned above, there exists a number of solutions of those equations that describe single, static, asymptotically-flat non-extremal black holes when we take $\kappa=-1$ [23, 24, 25], we can simply take those solutions and study them setting $\kappa=0$ or +1 in the transverse metric. Observe that one integration constant has been fixed to normalized the metric at spatial infinity, something we may not need to do in the $\kappa=0,+1$ cases, but the normalization could be changed at any moment, if necessary.

In what follows we are going to study the asymptotic behaviour of generic solutions $\left(U, \phi^{i}\right)$, normalized to describe single, static, asymptotically-flat non-extremal black holes for $\kappa=-1$ when we take the transverse metric with $\kappa=05$.

\section{Solutions with Lifshitz-like asymptotics}

Since we are going to use the metric functions $e^{-2 U}$ corresponding to charged, sphericallysymmetric, asymptotically-flat, non-extremal black-hole solutions, we start by reviewing their asymptotic behaviors at the outer $(+)$ and inner $(-)$ horizons 6 (placed, respectively, at $\tau=-\infty$ and $\tau=+\infty)$ and at spatial infinity $\tau=0$.

- The standard normalization of these asymptotically-flat black holes requires that

$$
\lim _{\tau \rightarrow 0^{-}} e^{-2 U}=1
$$

- When $\tau$ approaches the two horizons, $\tau \rightarrow \mp \infty$, the metric function behaves as

$$
e^{-2 U} \sim \frac{S_{ \pm}}{4 \pi r_{0}^{2}} e^{\mp 2 r_{0} \tau}
$$

where $S_{+}$(resp. $S_{-}$) is the entropy of the outer (resp. inner) horizon, which is assumed to be non-vanishing (which is equivalent to require regularity of the black-hole solution). If we use the spherically-symmetric transverse metric the spacetime metric approaches in these limits a product of a Rindler metric and a 2-sphere of area $4 S_{ \pm}$. Studying the Rindler metric by conventional methods one finds that the temperatures of the horizons $T_{ \pm}$obey the Smarr-like relation [29]

$$
r_{0}=2 S_{ \pm} T_{ \pm}
$$

- $e^{-2 U}$ vanishes for some value of $\tau_{\mathrm{s}} \in(0,+\infty)$ at which the physical singularity of the black-hole spacetime lies. We may also want to study the behaviour of $e^{-2 U}$ near this value of $\tau$ but we do not know of any general result on this respect. We will have to study each particular case separately.

\footnotetext{
${ }^{5}$ We leave the case $\kappa=+1$ for a future publication.

${ }^{6}$ Uncharged, static black holes only have outer horizon. The discussion of the behaviour of the metric function in the interior of the inner horizon does not apply to them.
} 
To find new solutions, we are going to plug black-hole metric functions in the general static metric Eq. (2.2) with the transverse metric Eq. (2.19) with $\kappa=0$, i.e. with Eq. (2.22) and Eq. (2.25). It is convenient to set $a=1 / r_{0}$ so no new length scale is introduced in the metric, which takes two possible forms:

$$
d s_{( \pm)}^{2}=e^{2 U} d t^{2}-e^{-2 U}\left[e^{ \pm 4 r_{0} \tau} r_{0}^{4} d \tau^{2}+e^{ \pm 2 r_{0} \tau} r_{0}^{2}\left(d \theta^{2}+d \phi^{2}\right)\right] .
$$

Asymptotic behaviour of $d s_{(-)}^{2}$ : Using the general properties of the metric function $e^{-2 U}$ described above it is easy to see that in the limit $\tau \rightarrow-\infty$ this metric behaves as

$$
d s_{(-)}^{2} \sim \frac{4 \pi r_{0}^{2}}{S_{+}} e^{2 r_{0} \tau} d t^{2}-\frac{S_{+}}{4 \pi r_{0}^{2}} e^{-2 r_{0} \tau}\left[e^{-4 r_{0} \tau} r_{0}^{4} d \tau^{2}+e^{-2 r_{0} \tau} r_{0}^{2}\left(d \theta^{2}+d \phi^{2}\right)\right] .
$$

The change of coordinates

$$
r \equiv e^{-r_{0} \tau}, \quad \tilde{t} \equiv \frac{4 \pi r_{0}^{2}}{S_{+}} t / r_{0}, \quad x^{1} \equiv \theta, \quad x^{2} \equiv \phi,
$$

brings the metric to the form

$$
d s_{(-)}^{2} \sim \frac{S_{+}}{4 \pi} r^{4}\left[r^{-6} d \tilde{t}^{2}-d r^{2}-d x^{i} d x^{i}\right]
$$

which is a hvLf metric of the form Eq. (1.1) with $z=4, \theta=6$ and radius

$$
\ell \sim r_{0}
$$

up to dimensionless factors (functions of the quotient $S_{+} / r_{0}^{2}$ ); observe that this asymptotic hvLf space lies in the class of Ricci flat hvLf spaces in Eq. (A.8).

The metric $d s_{(-)}^{2}$ is regular at $\tau=0$. Spatial infinity is not there because the radial distance between points with $\tau=0$ and points with $\tau<0$ is finite and not infinite, as in the black-hole case. For $\tau$ equal to a certain $\tau_{\mathrm{s}}, e^{-2 U}=0$ and the metric will be singular, as in the black-hole case. Finally, in the $\tau \rightarrow+\infty$ limit the metric is the product of Rindler spacetime times $\mathbb{R}^{2}$, which can be understood as a flat event horizon with the same temperature as that of the inner horizon of the associated black-hole solution.

Asymptotic behaviour of $d s_{(+)}^{2}$ : The analysis is completely analogous to the previous case: in the limit $\tau \rightarrow-\infty$ we find a flat event horizon whose temperature is that of the outer horizon of the associated black hole, there is a singularity at $\tau=\tau_{\mathrm{s}}$ and a hyperscaling Lifshitz metric in the $\tau \rightarrow+\infty$ limit. The Lifshitz radius is, once again, $\ell=r_{0}$.

\subsection{Examples}

The Schwarzschild black hole: This is the only uncharged, static, spherically-symmetric, black-hole solution of the class of theories we are considering and has only one horizon (the 
event horizon) at (conventionally) $\tau \rightarrow-\infty$ in these coordinates, which only cover the exterior. The metric function for the Schwarzschild black hole in these coordinates is

$$
e^{-2 U}=e^{2 M \tau}
$$

The spacetime metric $d s_{(-)}^{2}$ constructed with the Schwarzschild metric function takes the explicit form

$$
d s_{(-)}^{2}=e^{-2 M \tau} d t^{2}-e^{-2 M \tau} M^{4} d \tau^{2}-M^{2}\left(d \theta^{2}+d \phi^{2}\right) .
$$

In the coordinates

$$
e^{-M \tau} M \equiv r
$$

it reads

$$
d s_{(-)}^{2}=r^{2} d(t / M)^{2}-d r^{2}-M^{2}\left(d \theta^{2}+d \phi^{2}\right) .
$$

which is the product of a 2-dimensional Rindler spacetime $\left(\mathcal{R} i^{2}\right)$ with $\mathbb{R}^{2}$. The temperature of the flat horizon would be that of the Schwarzschild black hole $T \sim M^{-1}$. Observe that this is not just the asymptotic behaviour of the metric: the metric is everywhere identically $\mathcal{R} i^{2} \times \mathbb{R}^{2}$. As is well-known, this metric is just a wedge of the Minkowski spacetime which can be recovered by analytical extension of this metric.

Observe that the above metric makes sense for $\tau \in(-\infty,+\infty)$ or $r \in(0,+\infty)$ since as discussed above, there is not spatial infinity at $\tau=0$.

The metric $d s_{(+)}^{2}$ is in this case

$$
d s_{(+)}^{2}=e^{-2 M \tau} d t^{2}-e^{6 M \tau} M^{4} d \tau^{2}-e^{4 M \tau} M^{2}\left(d \theta^{2}+d \phi^{2}\right),
$$

and, in the coordinates

$$
e^{M \tau} \equiv r
$$

it takes the form

$$
d s_{(+)}^{2}=r^{-2} d(t / M)^{2}-r^{4} M^{2} d r^{2}-r^{4} M^{2}\left(d \theta^{2}+d \phi^{2}\right)=M^{2} r^{4}\left\{r^{-6} d t^{2}-d r^{2}-d \theta^{2}-d \phi^{2}\right\},
$$

which is the $z=4, \theta=6, \ell \sim M$ hvLf metric everywhere in the spacetime, and not just asymptotically. Yet again, this metric is defined for all values of $\tau$ or for all $r \in(0,+\infty)$.

The Reissner-Nordström black hole: The embedding of the Reissner-Nordström black hole in pure $\mathcal{N}=2, d=4$ supergravity (the supersymmetrization of the Einstein-Maxwell theory). The metric function of this solution in the $\tau$ coordinates is [23]

$$
e^{-2 U}=\left[\cosh r_{0} \tau-\frac{M}{r_{0}} \sinh r_{0} \tau\right]^{2}, \quad r_{0}^{2} \equiv M^{2}-|\mathcal{Z}|^{2},
$$

where

$$
\mathcal{Z}=\frac{1}{2} p-i q,
$$

is the central charge of pure $\mathcal{N}=2, d=4$ supergravity in the chosen conventions. 
It is evident that the asymptotic behaviour of the metrics $d s_{( \pm)}^{2}$ fits in the general case discussed above. Having the explicit form of the metric, we can also study the behaviour of the spacetime metric near the singularity at $\tau_{\mathrm{s}}$ at which $e^{-2 U}\left(\tau_{\mathrm{s}}\right)=0$. It is, however, easier to do it in the coordinates in which the metric function has the standard form

$$
e^{-2 U}=\frac{r^{2}}{\left(r-r_{+}\right)\left(r-r_{-}\right)}, \quad r_{ \pm}=M \pm r_{0}
$$

The coordinate transformation that relates these two forms of the metric function is

$$
r=-\left[\cosh r_{0} \tau-\frac{M}{r_{0}} \sinh r_{0} \tau\right]\left[\frac{\sinh r_{0} \tau}{r_{0}}\right]^{-1} .
$$

If we make this coordinate transformation in the full $d s_{( \pm)}^{2}$ metrics, they take the form

$$
d s_{( \pm)}^{2}=\frac{\left(r-r_{+}\right)\left(r-r_{-}\right)}{r^{2}} d t^{2}-\frac{r_{0}^{4} r^{2}}{\left(r-r_{ \pm}\right)\left(r-r_{\mp}\right)^{5}} d r^{2}-\frac{r_{0}^{2} r^{2}}{\left(r-r_{\mp}\right)^{2}}\left(d \theta^{2}+d \phi^{2}\right) .
$$

According to the general discussion, we should find the singularity in the extension of the metric beyond $\tau=0$ to positive values of $\tau$. This corresponds in these coordinates to values of $r$ "beyond $r=+\infty$ ". Thus, we define the coordinate $u \equiv 1 / r$ which overlaps with $r$ for $u>0$ and extends the metric for $u \leq 0$. In these coordinates the metric takes the form

$$
d s_{( \pm)}^{2}=\left(1-r_{+} u\right)\left(1-r_{-} u\right) d t^{2}-\frac{r_{0}^{4}}{\left(1-r_{ \pm} u\right)\left(1-r_{\mp} u\right)^{5}} d r^{2}-\frac{r_{0}^{2}}{\left(1-r_{\mp} u\right)^{2}}\left(d \theta^{2}+d \phi^{2}\right)
$$

and, in the $u \rightarrow-\infty$ limit it approaches the metric

$$
d s_{( \pm)}^{2}=r_{+} r_{-} u^{2} d t^{2}-\frac{r_{0}^{4}}{r_{ \pm} r_{\mp}^{5} u^{6}} d u^{2}-\frac{r_{0}^{2}}{r_{\mp}^{2} u^{2}}\left(d \theta^{2}+d \phi^{2}\right)
$$

which can be put in the hvLf form with $z=3, \theta=4$ (which implies $C(\theta, z)=0$ ) with the coordinate change $r^{\prime} \equiv 1 / u$ using rescaled the coordinates $\tilde{t} \equiv r_{ \pm} t / r_{0}^{2}, \rho \equiv r^{\prime} / r_{\mp}, x^{1} \equiv$ $\sqrt{r_{+} r_{-}} / r_{0} \theta, x^{2} \equiv \sqrt{r_{+} r_{-}} / r_{0} \phi$.

Observe that the two consecutive coordinate changes $r=1 / u, u=1 / r^{\prime}$ mean that we can get the same result taking the limit of the metric when $r$ approaches $r=0$ (which corresponds to the value $\tau=\tau_{\mathrm{s}}$ ) "from the left". In fact, the same result is obtained if we take the near-singularity limit from the right.

Summarizing, the interior of the inner horizon region $r<r_{-}$has, therefore, two boundaries, at $r=r_{-}$and at $r=0$. When the metric approaches $r=r_{-}$from the left, the metric $d s_{(+)}^{2}$ approaches a hvLf metric with $z=4$ and $\theta=6$ and the metric $d s_{(-)}^{2}$ approaches $\mathcal{R} i^{2} \times \mathbb{R}^{2}$, as we have seen before. When $r$ approaches $r=0$ the metric approaches a hvLf metric with $z=3, \theta=4$.

The fact that a hvLf metric can describe the near-singularity limit of a metric that has been obtained as a deformation of a regular black-hole metric is very suggestive. Observe that the 
deformed metric Eq. (3.20) differs from the standard Reissner-Nordström metric in factors of $\left(r-r_{ \pm}\right)$, which are irrelevant in the $r \rightarrow 0$ limit, and in the 2-dimensional metric $d \Omega_{\kappa}^{2}$ which has $\kappa=-1$ for the standard, spherically symmetric Reissner-Nordström black hole. In the next section we are going to see that there is a limit of the Reissner-Nordström black hole in which $d \Omega_{-1}^{2}$ approaches $d \Omega_{0}^{2}$. The near-singularity limit of this Reissner-Nordström black hole will be described by a hvLf metric with $z=3, \theta=4$.

\section{More hvLf metrics}

In this subsection we want to discuss some other ways of obtaining hvLf spacetimes.

\section{1 hvLf spaces from limiting procedures}

A 2-sphere looks locally (in small enough patches) like a plane. Thus, we can flatten $d \Omega_{-1}^{2}$ by looking at a small neighborhood of $\theta=\pi / 2$ and we can study near-horizon and near-singularity limits of standard, spherically-symmetric, black-hole solutions. The near-horizon limits will give, obviously, $\mathcal{R} i^{2} \times \mathbb{R}^{2}$ metrics (or $A d S_{2} \times \mathbb{R}^{2}$ metrics in the extremal cases).

Let us consider the near-singularity limit of the Reissner-Nordström black hole in a small patch around $\theta=\pi / 2$ :

$$
\begin{aligned}
d s^{2} & =\frac{\left(r-r_{+}\right)\left(r-r_{-}\right)}{r^{2}} d t^{2}-\frac{r^{2}}{\left(r-r_{+}\right)\left(r-r_{-}\right)} d r^{2}-r^{2}\left(d \theta^{2}+d \phi^{2}\right) \\
& \sim \frac{r_{+} r_{-}}{r^{2}} d t^{2}-\frac{r^{2}}{r_{+} r_{-}} d r^{2}-r^{2}\left(d \theta^{2}+d \phi^{2}\right),
\end{aligned}
$$

which can can be put in the hvLf form with $z=3, \theta=4$ and $\ell=\sqrt{r_{+} r_{-}}$with the coordinate change $r / \sqrt{r_{+} r_{-}} \rightarrow r, t / \sqrt{r_{+} r_{-}} \rightarrow t$.

We can also take the near-singularity limit of the Schwarzschild metric with negative mass in a neighborhood of $\theta=\pi / 2$

$$
\begin{aligned}
d s^{2} & =\left(1+\frac{2|M|}{r}\right) d t^{2}-\left(1+\frac{2|M|}{r}\right)^{-1} d r^{2}-r^{2}\left(d \theta^{2}+d \phi^{2}\right) \\
& \sim \frac{2|M|}{r} d t^{2}-\frac{r}{2|M|} d r^{2}-r^{2}\left(d \theta^{2}+d \phi^{2}\right),
\end{aligned}
$$

which can be put in the hvLf form with $z=4, \theta=6$ and $\ell=|M| / 2$ with the coordinate change $2 r /|M| \rightarrow \rho^{2}, 4 t /|M| \rightarrow t$.

\subsection{Supersymmetric hvLf spaces from smearing}

As was mentioned briefly in Section 2 , the extremal limit $\left(r_{0} \rightarrow 0\right)$ of the 4-dimensional metric describes a single static black hole and the natural question, one we have been ignoring, is what happens in the case $\kappa=0$. 
The first thing that changes is the asymptotic behaviour of $e^{-2 U}$, which for an extremal black hole reads

$$
\lim _{\tau \rightarrow-\infty} e^{-2 U}=\frac{S}{\pi} \tau^{2}
$$

where $S$ is the entropy of the black hole. The second thing is that the extremal limit of $W_{\kappa}^{ \pm}$is just the constant $a$ which has the dimension of inverse length, whence the 4-dimensional metric becomes

$$
d s_{0}^{2}=e^{2 U} d t^{2}-e^{-2 U}\left[d\left(a^{-2} \tau\right)^{2}+d \vec{x}^{2}\right],
$$

where we have defined $x^{1}=\theta / a$ and $x^{2}=\phi / a$. It is straightforward to see that in the region $\tau \rightarrow-\infty$ this leads to a hvLf space with $\theta=4$ and $z=3$. Similarly to what happens in the Schwarzschild black hole case in Section 3.1, one can see that the extremal RN black hole of electrical charge $q$, which has $e^{-U}=1-\frac{|q|}{\sqrt{2}} \tau$, is this asymptotic hvLf.

Now we are going to see that this solution is just a particular case of a very wide class of solutions with hvLf asymptotics.

One of the most interesting features of the extremal RN black hole is that it is supersymmetric in pure $N=2, d=4$ supergravity. As is well known, the most general supersymmetric static solution of this theory can be written, using Cartesian coordinates in the transverse space $\vec{y}_{3} \equiv$ $\left(y^{1}, y^{2}, y^{3}\right)$ as

$$
d s_{\text {susy }}^{2}=e^{2 U} d t^{2}-e^{-2 U} d \vec{y}_{3}^{2},
$$

where the metric function has the form?

$$
e^{-2 U}=\frac{1}{2}\left(H^{0}\right)^{2}+2\left(H_{0}\right)^{2},
$$

where $H_{0}$ and $H^{0}$ are two real harmonic functions in the flat transverse space which satisfy the staticity constraint

$$
H^{0} \partial_{m} H_{0}-H_{0} \partial_{m} H^{0}=0, m=1,2,3 .
$$

In these coordinates, the standard, spherically symmetric $(\kappa=-1)$, purely electric extremal RN black hole corresponds to the choice of harmonic functions

$$
H^{0}=0, \quad H_{0}=1+\frac{1}{\sqrt{2}} \frac{|q|}{\left|\vec{y}_{3}\right|} .
$$

However, other choices (usually discarded when one is only interested in black holes) are possible and are also supersymmetric. For instance, one can consider harmonic functions that depend on only one of the transverse coordinates, say $y^{3} \equiv \rho$. This corresponds, physically, to the smearing of the spherically-symmetric solution in the $\left(y^{1}, y^{2}\right)$ plane and, mathematically, to the substitution of the factor $1 / r$ by $\rho$ in all the harmonic functions of the spherically-symmetric solution. The staticity constraint Eq. (4.7) is automatically satisfied is it was in the sphericallysymmetric solution.

From the the extremal RN solution, this choice gives the new smeared solution

$$
d s^{2}=\frac{1}{2}\left(H_{0}\right)^{-2} d t^{2}-2\left(H_{0}\right)^{2}\left[d \rho^{2}+d y^{i} d y^{i}\right], \quad H_{0}=1+\frac{1}{\sqrt{2}}|q| \rho,
$$

\footnotetext{
${ }^{7}$ We use the conventions of Ref. [23].
} 
and this solution is identical to the $\kappa=0$ solution in Eq. 4.48 with $\tau=-\rho$. Furthermore, the $z \rightarrow \infty$ limit, which gives the $\theta=3, z=4$ hvLf space corresponds to the choice

$$
H_{0}=\frac{1}{\sqrt{2}}|q| \rho
$$

and, therefore, it is an exact, supersymmetric solution.

Once this connection between hvLf metrics and smeared supersymmetric black holes of $N=$ $2, d=4$ supergravity has been established, we can systematically construct supersymmetric hvLf metrics using the well-known systematic procedure to construct all the supersymmetric black hole solutions of any $N=2, d=4$ supergravity coupled to vector supermultiplets [31, 32, 33, 34] and choosing harmonic functions that depend on only one coordinate in transverse space. The $\rho \rightarrow \infty$ limit is the same in all the cases (namely a $\theta=3, z=4 \mathrm{hvLf}$ spacetime), provided that the original, spherically-symmetric solution has a regular near-horizon limit. The scalar fields, which have non-trivial profiles in the smeared solutions, become constant in the $\rho \rightarrow \infty$ limits, just as they do in the black-hole near-horizon limits.

There are, however, more possibilities, if we start from supersymmetric black holes with singular horizon. A good example is provided by the supersymmetric D0-D4 black holes embedded in the $S T U$ model [35, 36, 37] . After the smearing, the three complex scalars $Z^{i}, i=1,2,3$ and metric function of these solutions are given by

$$
\begin{gathered}
Z^{i}=-4 i e^{2 U} H_{0} H^{i}, \\
e^{-2 U}=4 \sqrt{H_{0} H^{1} H^{2} H^{3}},
\end{gathered}
$$

where the four harmonic functions $H_{0}, H^{1}, H^{2}, H^{3}$ are

$$
\begin{aligned}
& H_{0}=s_{0}\left\{a_{0}+\frac{1}{\sqrt{2}} \frac{\left|q_{0}\right|}{\left|\vec{y}_{3}\right|}\right\}, \\
& H^{i}=s^{(i)}\left\{a^{(i)}+\frac{1}{\sqrt{2}} \frac{\left|p^{(i)}\right|}{\left|\vec{y}_{3}\right|}\right\},
\end{aligned}
$$

where $a_{0}, a^{i}$ are constants related to the asymptotic $(r \rightarrow \infty)$ values of the scalars, $q_{0}, p^{i}$ are electric and magnetic charges and $s_{0}, s^{i}$ are the signs of those charges. Only two sets of signs of charges lead to supersymmetric and regular black holes: all charges positive or negative. In particular, none of these charges can vanish.

The associated smeared solutions are given by Eqs. (4.11) and (4.12) with the harmonic functions given by

$$
\begin{aligned}
& H_{0}=s_{0}\left\{a_{0}+b_{0} \rho\right\}, \\
& H^{i}=s^{(i)}\left\{a^{(i)}+b^{(i)} \rho\right\} .
\end{aligned}
$$

\footnotetext{
${ }^{8}$ Observe that, in the non-extremal case, we cannot view the $\kappa=0$ solutions as the smearing of $\kappa=-1$ solutions.

${ }^{9}$ Again, we use the notation and conventions of Ref. [23] where the details can be found.
} 
The constants $b_{0}, b^{i}$, which we can take to be positive, are related to electric and magnetic fluxes. The staticity condition Eq. (4.7) is satisfied for any values of the constants and, in particular, we can take any number of them to vanish.

When all the $b_{0}, b^{i}$ constants are different from zero, we can take all the $a_{0}, a^{i}$ to vanish or take the $\rho \rightarrow \infty$ limit. In both cases $e^{-2 U}=4 \sqrt{b_{0} b^{1} b^{2} b^{3}} \rho^{2}$ and we get a $\theta=3, z=4 \mathrm{hvLf}$ spacetime with constant scalars.

When one of them $\left(b_{0}\right.$, for instance) vanishes we must keep $a_{0} \neq 0$, and we get

$$
Z^{i}=-i \frac{a_{0} b^{i}}{\sqrt{a_{0} b^{1} b^{2} b^{3}}} \rho^{-1 / 2}, \quad e^{-2 U}=4 \sqrt{a_{0} b^{1} b^{2} b^{3}} \rho^{7 / 2}
$$

which is a $\theta=7 / 2, z=5 / 2 \mathrm{hvLf}$ spacetime, now with non-trivial scalars. Other choices of vanishing constant $b$ lead to different scalar profiles by the same $\theta$ and $z$.

It is easy to see that, for $n=0, \cdots, 4$ non-vanishing constants $b$, one gets a hvLf spacetime with $\theta=2+n / 2$ and $z=1+n / 2$ and various scalar profiles. Perhaps not surprisingly $C_{(\theta, z)}=(4-n) / n$ and only vanishes for $n=4$.

\subsection{Higher dimensional generalization}

In ref. [30] the FGK formalism was Generalised to higher dimensional cases, and it is only natural to consider the higher dimensional generalizations of the results presented in the foregoing sections, starting off by the ones in Section 2 , the $D$-dimensional generalization of the FGK metric reads

$$
d s^{2}=e^{2 U} d t^{2}-e^{-\frac{2}{d-1} U}\left[\frac{d \rho^{2}}{(d-1)^{2} W_{\kappa}^{2 d /(d-1)}}+\frac{\mathrm{h}_{i j} d x^{i} d x^{j}}{W_{\kappa}^{2 /(d-1)}}\right],
$$

where $\mathrm{h}$ is the metric of a $d$-dimensional Riemannian Einstein space; this metric is normalized such that

$$
R(\mathrm{~h})_{i j}=(d-1) \kappa \mathrm{h}_{i j} .
$$

The normalization is such that a $d$-sphere with the round metric has $\kappa=-1$.

A so-so calculation then shows that the conditions for the resulting FGK equations of motion to be $\kappa$ as well as $W_{\kappa}$ independent, are

$$
W_{\kappa} \ddot{W}_{\kappa}-\dot{W}_{\kappa}^{2}=\kappa \quad \text { and } \quad \ddot{W}_{\kappa}=\mathcal{B}^{2} W_{\kappa} ;
$$

$\mathcal{B}$ plays the rôle of the $D$-dimensional non-extremality constant which on dimensional grounds can be written as $r_{0}^{d-1}$. The solutions to the conditions (4.18) are

$$
W_{-1}=\frac{\sinh (\mathcal{B} \rho)}{\mathcal{B}}, W_{0}^{ \pm}=a e^{\mp \mathcal{B} \rho} \quad \text { and } \quad W_{1}=\frac{\cosh (\mathcal{B} \rho)}{\mathcal{B}} .
$$

By looking at the, in general, fractional powers of $W$ that appear in the metric (4.16), we see that in contradistinction to the 4-dimensional case, the putative horizon lies at $\rho \rightarrow \infty$ which means that the near-horizon behaviour for a non-extremal black hole implies

$$
\lim _{\rho \rightarrow \infty} e^{U} \sim e^{-\mathcal{B} \rho}
$$


The above means that given a solution to a $D$-dimensional FGK system, we can as before deform the $\kappa=-1$ solution as in Section 2, and obtain new solutions with properties similar to the ones encountered in the foregoing sections. For example, concerning the $\rho \rightarrow \infty$ behaviour of the metric we see that

The $W_{0}^{+}$case: $\quad$ In this case the $\rho \rightarrow \infty$ spacetime is hvLf with

$$
\theta=\frac{d(d+1)}{d-1} \quad \text { and } \quad z=\frac{2 d}{d-1},
$$

which, as one can see from Eq. (A.8), corresponds to the Ricci flat hvLf spaces.

The $W_{0}^{-}$case: Together with the condition (4.20), we see that the resulting $\rho \rightarrow \infty$ spacetime is a Rindler wedge times $\mathbb{R}^{d}$.

In the $\kappa=-1$ case, the validity of the $\rho$-coordinate, i.e. $\rho \in[0, \infty)$ is principally determined by $W_{-1}$ and one imposes conditions on $e^{U}$ in order to obtain metrics describing the spacetime outside the outer horizon. In particular, the zero of $W_{-1}$ at $\rho=0$, together with the regularity of $e^{U}$ there, allows for the identification of $\rho=0$ with asymptotic spacetime. $W_{0}$ is, however, an all-together different beast and the naive validity of the coordinate, i.e. $\rho \in[0, \infty)$, can be extended till one encounters a zero or a pole in $e^{U}$; the former signaling a horizon, the latter a singularity. Let us illustrate this point with

The 5-dimensional STU model: The FGK equations for the 5-dimensional STU model are completely separable, whence the full analytical solution is known. The general solution satisfying Eq. (4.20) and having constant scalars in the limit $\rho \rightarrow \infty$ is given by (see e.g. [24, 27])

$$
e^{-3 U}=\frac{\left|\mathrm{q}_{1} \mathrm{q}_{2} \mathrm{q}_{3}\right|}{\mathcal{B}^{3}} \sinh \left(\alpha_{1}+\mathcal{B} \rho\right) \sinh \left(\alpha_{2}+\mathcal{B} \rho\right) \sinh \left(\alpha_{3}+\mathcal{B} \rho\right),
$$

where the $q$ are the electrical charges and the $\alpha$ 's are some real constants; in the $\kappa=-1$ case they are chosen such that $U(\rho=0)=1$ and one obtains a Minkowski space with the regular normalization. In the $\kappa=0$ case, however, the point $\rho=0$ is not asymptotic and there is therefore no need to impose said condition on the $\alpha$ 's. In fact, let $0<\alpha_{1} \leq \alpha_{2} \leq \alpha_{3}$, then we can extend the definition of $\rho$ to the point $\rho_{s}=-\alpha_{1} / \mathcal{B}$, where we have added a subscript to highlight the fact that at that point we're facing a curvature singularity.

As in Section 3.1 we can consider the near-singularity metric: in general we will find a hvLf space and the characteristic parameters $(\theta, z)$ will depend on the order of the zero of $e^{-3 U}$ in Eq. (4.22). Denoting this number by $p$, whence $p=1,2$ or 310 we see that the near-singularity hvLf is given by

$$
\theta=3+\frac{\mathrm{p}}{2}, \quad z=1+\frac{\mathrm{p}}{2} \quad \text { whence } C_{(\theta, z)}=\frac{2(3-\mathrm{p})}{\mathrm{p}} \geq 0
$$

\footnotetext{
${ }^{10}$ To wit: $\mathrm{p}=1$ implies $\alpha_{2}>\alpha_{1}, \mathrm{p}=2$ means $\alpha_{3}>\alpha_{2}=\alpha_{1}$ and $\mathrm{p}=3$ means $\alpha_{3}=\alpha_{2}=\alpha_{1}$. Let us in passing observe that the case $\mathrm{p}=3$ corresponds to the deformation of the 5-dimensional Reissner-Nordström black hole.
} 
and, furthermore, the null energy condition A.7 is always satisfied.

In higher dimensions we can also construct hvLf solutions by smearing extremal, supersymmetric black-hole solutions. The procedure is entirely similar to the one followed in four dimensions.

In a higher-dimensional context, it is natural to consider the following brane-like generalization of the hvLf metric (1.1)

$d s_{d+2}^{2}=\ell^{2} r^{-2(d-\theta) / d}\left[r^{-2(z-1)}\left(d t^{2}-d y^{a} d y^{a}\right)-d r^{2}-d x^{i} d x^{i}\right], \quad a=1, \cdots, p, \quad i=1, \cdots d$.

The $p=0$ case is the original hvLf metric and a metric with $d=0$ and $p=\neq 0$ can be rewritten as a $p=0, d \neq 0$ by a coordinate change.

It should come as no surprise that we can obtain metrics of this kind by smearing extremal supersymmetric $p$-brane metrics. As an example, consider the 10-dimensional $\mathrm{D} p$-brane solutions in the Einstein frame

$$
\begin{aligned}
d s^{2} & =H^{\frac{p-7}{8}}\left[d t^{2}-d \vec{y}_{p}^{2}\right]-H^{\frac{p+1}{8}} d \vec{x}_{8-p}^{2}, \\
C_{(p+1) t y^{1} \ldots y^{p}} & = \pm e^{-\phi_{0}}\left(H^{-1}-1\right), \\
e^{-2 \phi} & =e^{-2 \phi_{0}} H^{\frac{p-3}{2}} .
\end{aligned}
$$

In all cases, we can take 11

$$
H \sim \rho
$$

and put the metric in the form

$$
\begin{aligned}
d s^{2} & \sim \rho^{\frac{p+1}{8}}\left\{\rho^{-1}\left[d t^{2}-d \vec{y}_{p}^{2}\right]-d \rho^{2}-d \vec{x}_{8-p}^{2}\right\}, \\
C_{(p+1) t y^{1 \ldots y}} & =\sim \rho^{-1}, \\
e^{-2 \phi} & \sim \rho^{\frac{p-3}{2}},
\end{aligned}
$$

which is of the above form with $p=p, z=3 / 2$ and $\theta=(8-p)(p+17) / 16$ for $p<8$. The case $p=0$ (the D0-brane) is a standard hvLf metric with $d=8, \theta=8.5$ and $z=3 / 2$, which satisfies the null energy condition (A.7) but does not avoid the null curvature singularity in the IR region $(\rho \rightarrow \infty)$. The string coupling constant reads $e^{\phi}=r^{3 / 4}$, which goes to zero in the UV. The case $p=8$, after a change of coordinates $\varrho \equiv \rho^{3 / 2}$ is also a standard hvLf metric $(p=0)$ with $d=8$, $\theta=25 / 3$ and $z=1$ which also satisfies the null energy condition A.7 but is singular in the IR region $(r \rightarrow \infty)$.

\section{Discussion}

In this paper we have shown that hvLf metrics appear in many near-horizon and near-singularity limits of well-known solutions or solutions that one can easily construct by deforming them.

\footnotetext{
${ }^{11}$ In the $p=8$ case there is no smearing involved, since there is only one transverse dimension.
} 
The abundance of examples seems to suggest that hvLf metrics capture the behavior of many spacetimes near certain curvature singularities; in particular near timelike singularities such as those of the extremal RN black hole or the Schwarzschild solution with negative mass.

Since some hvLf are holographically related to some well-known QFTs, this apparently general property suggests the very attractive possibility of finding holographically related QFTs that can describe those classical curvature singularities, at least in some regime. Finding quantum systems with the right values of $z$ and $\theta$ may be difficult, or impossible, though. More work is needed to see if this possibility can be realized.

\section{Acknowledgments}

The authors would like to thank E. Ó Colgáin and S. Kachru for stimulating discussions. WC and CSS would like to thank the Stanford Institute for Theoretical Physics for its hospitality; likewise, PM would like to thank the Instituto de Física Teórica its hospitality. This work has been supported in part by the Spanish Ministry of Science and Education grant FPA2009-07692, the Principáu d'Asturies grant IB09-069, the Comunidad de Madrid grant HEPHACOS S2009ESP1473, and the Spanish Consolider-Ingenio 2010 program CPAN CSD2007-00042. The work of PM has been supported by the Ramón y Cajal fellowship RYC-2009-05014. The work of PB and CSS has been supported by the JAE-predoc grants JAEPre 201100452 and JAEPre 201000613. TO wishes to thank M.M. Fernández for her constant support.

\section{A Some properties of the hvLf metrics}

The hvLf metric (1.1) is spatially homogeneous and covariant under the scale transformations

$$
x_{i} \rightarrow \lambda x_{i}, t \rightarrow \lambda^{z} t, r \rightarrow \lambda r, d s_{d+2}^{2} \rightarrow \lambda^{2 \theta / d} d s_{d+2}^{2},
$$

where $\lambda$ is a dimensionless parameter. Observe that this means that the Lifshitz radius $\ell$ is only defined up to dimensionless factors. The Ricci tensors of metrics (1.1) are given by

$$
\begin{aligned}
R_{t t} & =\frac{(d z-\theta)(d+z-\theta)}{d r^{2 z}} \\
R_{r r} & =\frac{(d+z) \theta-d\left(z^{2}+d\right)}{d r^{2}} \\
R_{i j} & =\frac{(\theta-d)(d+z-\theta)}{d r^{2}} \delta_{i j} .
\end{aligned}
$$

This geometry generically suffers from a null curvature singularity at $r=\infty$ except for a specific set of parameter values. The singularity exists even though all curvature invariants remain finite. The tidal forces diverge as [7]

$$
C_{(\theta, z)} r^{2 C_{(\theta, z)}+d}, \quad C_{(\theta, z)}=\frac{d(z-1)-\theta}{d-\theta}
$$


where we have restricted to $C_{(\theta, z)}>0$ for which the singularity is a null curvature singularity as surfaces of constant $r$ become null as $r$ goes to infinity. We distinguish several cases:

- For $\theta=0$ we simply get the result in [19] which is appropriate for Lifshitz scaling. Ways for resolving the null curvature singularities have been presented in [20, 21].

- The case of $\theta=0$ and $z=1$ is the non-singular result of pure AdS.

- There are non-singular results for

$$
C_{(\theta, z)}=0, \text { or } C_{(\theta, z)}+1 \leq 0
$$

The null energy condition in the bulk gives the conditions

$$
C_{(\theta, z)} \geq 0, \quad(z-1)(d+z-\theta) \geq 0,
$$

which rules out the non-singular condition $C_{(\theta, z)}+1 \leq 0$ and leaves the condition $C_{(\theta, z)}=0$.

There is a class of Ricci-flat hvLf spaces: they are characterized by

$$
\theta=\frac{d(d+1)}{d-1} \quad \text { and } \quad z=\frac{2 d}{d-1} \rightarrow C_{(\theta, z)}=0
$$

These spaces always solve the null energy condition and are regular in the IR interior $(r \rightarrow \infty)$.

\section{References}

[1] S. A. Hartnoll, Class. Quant. Grav. 26, 224002 (2009) [0 903.3246 ].

[2] C. P. Herzog, J. Phys. A A 42, 343001 (2009) [090 4 . 1975].

[3] J. McGreevy, Adv. High Energy Phys. 2010 (2010) 723105 [0 909 . 0518].

[4] C. Charmousis, B. Gouteraux, B. S. Kim, E. Kiritsis and R. Meyer, JHEP 1011, 151 (2010) [1005.4690].

[5] L. Huijse, S. Sachdev and B. Swingle, Phys. Rev. B 85, 035121 (2012) [1112 . 0573].

[6] X. Dong, S. Harrison, S. Kachru, G. Torroba and H. Wang, JHEP 1206, 041 (2012) [1201.1905].

[7] E. Shaghoulian, JHEP 1205, 065 (2012) [1112 . 2702].

[8] N. Ogawa, T. Takayanagi and T. Ugajin, JHEP 1201, 125 (2012) [1111. 1023].

[9] E. Perlmutter, JHEP 1206, 165 (2012) [1205 . 02 42].

[10] K. Narayan, Phys. Rev. D 85, 106006 (2012) [1202 . 5935]. 
[11] M. Ammon, M. Kaminski and A. Karch, 1207.1726.

[12] E. Witten, Adv. Theor. Math. Phys. 2 (1998) 253 [hep-th/9802150].

[13] E. Witten, Adv. Theor. Math. Phys. 2 (1998) 505 [hep-th/9803131].

[14] I. Amado and A. F. Faedo, JHEP 1107, 004 (2011) [1105 . 4862].

[15] L. Barclay, R. Gregory, S. Parameswaran, G. Tasinato and I. Zavala, JHEP 1205, 122 (2012) [1203.0576].

[16] W. Chemissany and J. Hartong, Class. Quant. Grav. 28, 195011 (2011) [1105. 0612 ].

[17] N. Iizuka, N. Kundu, P. Narayan and S. P. Trivedi, JHEP 1201, 094 (2012) [1105.1162].

[18] M. Alishahiha, E. Ó Colgáin, H. Yavartanoo, preprint IPM/P-2012/050, FPAUO-12/12.

[19] G. T. Horowitz and B. Way, Phys. Rev. D 85, 046008 (2012) [1111. 1243 ].

[20] S. Harrison, S. Kachru and H. Wang, 1202 . 6635.

[21] N. Bao, X. Dong, S. Harrison and E. Silverstein, 1207.0171.

[22] S. Ferrara, G. W. Gibbons and R. Kallosh, Nucl. Phys. B 500 (1997) 75 [hep-th/9702103].

[23] P. Galli, T. Ortín, J. Perz and C. S. Shahbazi, JHEP 1107, 041 (2011) [1105.3311].

[24] T. Mohaupt and O. Vaughan, JHEP 1207 (2012) 163 [1112 . 2876].

[25] P. Meessen, T. Ortín, J. Perz and C. S. Shahbazi, Phys. Lett. B 709 (2012) 260 [1112.3332].

[26] M. Hübscher, P. Meessen and T. Ortín, Nucl. Phys. B 759 (2006) 228 [hep-th/0606281].

[27] P. Meessen, T. Ortín, J. Perz and C. S. Shahbazi, JHEP 1209 (2012) 001 [12 04 . 050 7].

[28] A. de Antonio Martín, T. Ortín and C. S. Shahbazi, JHEP 1205 (2012) 045 [1203 . 0260].

[29] G. W. Gibbons, R. Kallosh, B. Kol, Phys. Rev. Lett. 77 (1996) 4992-4995. [hep-th/9607108].

[30] P. Meessen and T. Ortín, Phys. Lett. B 707 (2012) 178 [1107. 5454 ].

[31] K. Behrndt, D. Lüst, W. A. Sabra, Nucl. Phys. B510 (1998) 264-288. [hep-th/9705169].

[32] G. L. Cardoso, B. de Wit, J. Käppeli, T. Mohaupt, JHEP 0012 (2000) 019. [hep-th/0009234]. 
[33] F. Denef, JHEP 0008 (2000) 050 [hep-th / 000504 9].

[34] P. Meessen, T. Ortín, Nucl. Phys. $B 749$ (2006) 291-324. [hep-th / 0603099 ].

[35] M. J. Duff, J. T. Liu and J. Rahmfeld, Nucl. Phys. B 459 (1996) 125 [hep-th/9508094].

[36] K. Behrndt, R. Kallosh, J. Rahmfeld, M. Shmakova and W. K. Wong, Phys. Rev. D 54 (1996) 6293 [hep-th/9608059].

[37] S. Bellucci, S. Ferrara, A. Marrani and A. Yeranyan, Entropy 10 (2008) 507 [080 7 . 3503].

[38] M. Alishahiha, E. Ó Colgáin, H. Yavartanoo, Preprint IPM/P-2012/050, FPAUO-12/10. 